\title{
Research related to spinal cord management is international
}

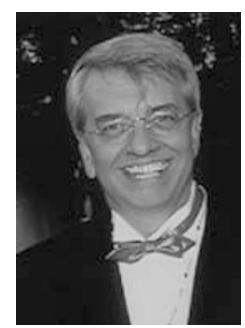

JJ Wyndaele, Editor

Antwerp University Hospital, Antwerp, Belgium

E-mail: spinalcord@uza.be

Dear Spinal Cord reader,

This is the final issue of Spinal Cord for 2009. We have had a good year, with many interesting submissions and increasing interest from an international readership. In October, the ISCOS Annual Scientific meeting in Florence was very successful and enjoyable. We are looking forward to publishing the interesting studies presented. Various changes to the management and content of Spinal Cord were announced during the Annual Editorial Board meeting; for example, changes in board membership, the introduction of editorial comments linked to specific manuscripts and co-authorship of editorial page messages. All who help to further develop Spinal Cord into the forum of excellence for spinal cord management and research, are greatly thanked.

In the review article, Sakakibara et al. assessed the psychometric properties of depression and anxiety instruments used with spinal cord injury (SCI) populations. Given that the reliability and validity findings range for the most part from adequate to excellent, and the large amount of work to develop cutoff scores specific for SCI populations, the authors find at present no need to develop SCI-specific instruments. Because the psychometric properties of one measure do not clearly stand out, the authors find it difficult to recommend the use of one over another. Overall, more psychometric data is needed, and if the instruments are to be used to evaluate treatment outcomes or change over time, responsiveness data is also required. Administering the instruments in tandem with each other and with clinical diagnostic interviews would provide valuable information, as would comparison of results to normative data specific to persons with SCI.

House et al. examined caregivers' perspectives on the effectiveness of rehabilitative support experienced by youth with spinal cord injury (SCI) during acute rehabilitation and after community reintegration in terms of their community participation. Findings from this study can be used to help professionals tailor rehabilitation programs to better meet the needs of youth with SCI and their families, thereby increasing chances of successful reintegration back into their communities.

Chhabra et al. determined the safety and feasibility of autologous olfactory mucosal transplantation into the spinal cord in patients with chronic spinal cord injury (CSCI) in a pilot study at the Spinal Injury Centre, New Delhi. In the five patients included, they found the procedure relatively safe and feasible in American Spinal Injury Association (ASIA) Impairment Scale (AIS) A participants with thoracic level injuries at 18 month follow up. No efficacy could be demonstrated that could be attributed to the procedure. The authors state that it might not be possible to conclude regarding the efficacy of the procedure due to limitations of the study.

Finnerup et al. evaluated the efficacy of the anticonvulsant levetiracetam in patients with spinal cord injury at- and below-level pain and secondarily the effect on spasm severity. No relief of neuropathic pain or spasm severity was found.

Xue-Feng Shen et al. developed a modified ferric tannate method for the study of spinal cord injury that can visualize the blood vessels and be compatible with hematoxylin and eosin (HE) and immunohistochemical techniques, and found it superior to the original one.

Chaoquin Ye et al. determined the characteristics of sports and recreation related (SR-related) spinal cord injuries (SCIs) in Beijing. SCI as a result of diving accidents was most commonly seen among young, male adults. There was a significant increase in sports injuries other than diving in later years. Successful prevention programs from other countries have been transferred to Beijing in recent years, so safety improvement is expected in the years to come. 
Warwick et al. found that MRI correlates well with CT in identifying vertebral fracture union and non union. They suggest that where imaging is indicated in the assessment of vertebral body fracture healing MRI can be used routinely, with CT reserved for problematic or inconclusive cases.

Several studies have demonstrated neurological prostatic dysfunction in patients with SCI, as confirmed by low levels of seminal prostate specific antigen (PSA), which is used as a parameter of gland activity. Alexandrino et al. determined whether SCI causes seminal citrate concentration in a comparison between patients and healthy men. Patients with SCI have a significant reduction of seminal citrate as a consequence of neurological dysfunction of the prostate.

Enjoy reading the case reports

From the editorial office, we wish you a Happy New Year and look forward to further cooperation in 2010.

Spinal Cord (2009) 47, 839-840; doi:10.1038/sc.2009.162 